SPIN-02/46

ITP-UU-02/70

ROM2F $/ 2002 / 33$

hep-th/0212255

\title{
Calabi-Yau Fourfolds with Flux and Supersymmetry Breaking
}

\author{
Marcus Berg, 巴† Michael Haack P $^{\dagger}$ and Henning Samtleben $\}^{\dagger * *}$ \\ † Dipartimento di Fisica \\ Universitá di Roma, Tor Vergata \\ 00133 Rome, Italy \\ * Institute for Theoretical Physics $\&$ Spinoza Institute \\ Utrecht University, Postbus 80.195 \\ 3508 TD Utrecht, The Netherlands
}

\begin{abstract}
In Calabi-Yau fourfold compactifications of M-theory with flux, we investigate the possibility of partial supersymmetry breaking in the three-dimensional effective theory. To this end, we place the effective theory in the framework of general $N=$ 2 gauged supergravities, in the special case where only translational symmetries are gauged. This allows us to extract supersymmetry-breaking conditions, and interpret them as conditions on the 4-form flux and Calabi-Yau geometry. For $N=2$ unbroken supersymmetry in three dimensions we recover previously known results, and we find a new condition for breaking supersymmetry from $N=2$ to $N=1$, i.e. from four to two supercharges. An example of a Calabi-Yau hypersurface in a toric variety that satisfies this condition is provided.
\end{abstract}

\footnotetext{
${ }^{1}$ e-mail: berg@roma2.infn.it

${ }^{2}$ e-mail: haack@roma2.infn.it

${ }^{3}$ e-mail: h.samtleben@phys.uu.nl
} 


\section{Introduction}

In any attempt to make contact between string/M-theory and phenomenology, one has to explain why the world looks effectively four-dimensional. One possibility would be that the extra dimensions are compactified on a manifold too small to be noticed at low energies. Then, in turn, one has to face the problem that every parameter in the metric of the internal space shows up as a massless scalar field in the effective theory - contradicting experiment. One solution to this problem that has recently attracted renewed interest consists in turning on internal background values for $p$-form field-strengths in the higher-dimensional theory. This leads to a gauging of the supergravity in the low energy effective action and, quite generically, a potential for the scalars of the "no-scale" type is induced.f Thus at least some of the scalar fields become massive.

From a phenomenological point of view, compactifications with unbroken $N=1$ supersymmetry in four dimensions seem most appealing in attempts to approach questions like the hierarchy or cosmological constant problem. At the same time, only $N=1$ supersymmetry is consistent with a chiral particle spectrum. A scenario leaving this amount of supersymmetry in the context of flux compactifications was put forward in [1]. There, orientifolds of type IIB Calabi-Yau compactifications with 3-form flux were studied. f However, due to the presence of O3-planes (and possibly D3-branes), the derivation of the effective theory using the Kaluza-Klein procedure is not straightforward.

In this note we discuss an alternative, related setting: effective theories that are related to the type IIB orientifolds with 3 -form flux, but have a more direct description as Kaluza-Klein compactifications. In particular, we consider elevendimensional supergravity on Calabi-Yau fourfolds with 4-form flux. Such compactifications preserve $N=2$ supersymmetry in three dimensions, which is the same amount as $N=1$ in four dimensions. The precise relation of these fourfold compactifications to IIB orientifolds can be established as follows. If the Calabi-Yau fourfold is elliptically fibered, the F-theory limit relates the fourfold compactification to type IIB compactified on the base of the elliptic fibration [10. At particular loci of the moduli space the type IIB compactifications have a dual description as type IIB orientifolds [1]. Furthermore, the 3 -form flux of the type IIB compactifications stems from the lift of the original 4-form flux [12, 13].

One of the main questions in fourfold compactifications that we want to address is whether it is possible to break supersymmetry spontaneously from $N=2$ to $N=1$, i.e. from four to two supercharges. Our strategy to answer this question is the following. We will argue that the effective theories from compactifications of eleven-dimensional supergravity on Calabi-Yau fourfolds with 4-form flux are

\footnotetext{
${ }^{4}$ However, stringy $\alpha^{\prime}$-corrections spoil the no-scale structure of the potential [1, 2].

${ }^{5}$ For a thorough introduction to orientifolds see [3] and the references therein. The scalar potential was also investigated in [4, 5, 6, 7, 8, 9], however in the $N=2$ context of Calabi-Yau compactifications without orientifolding.
} 
special cases of three-dimensional gauged supergravities, where some Peccei-Quinn (PQ) symmetries are gauged (in the case of the fourfold compactifications these PQ symmetries stem from the gauge symmetries of the 3-form). Using the general results of [14] about three-dimensional gauged supergravities it is then straightforward to find a condition for partial supersymmetry breaking.

The effective theories from Calabi-Yau fourfold compactifications of elevendimensional supergravity with 4-form flux were previously considered in [15, 16, 12, 13, 17, 18, 19, 2]. In [15], conditions on the flux for preserving $N=2$ supersymmetry were derived. In particular, only a primitive flux of type $(2,2)$ preserves $N=2$. It was argued in [12 that this condition can be encoded in two superpotentials, whereas it was shown in 18 that the potential resulting from a Kaluza-Klein reduction involves one superpotential and a further contribution expressed via a real function closely related to the proposed second superpotential of [12]. We show that the correctness of the potential found from Kaluza-Klein reduction in [18 is confirmed by the general $N=2$ gauged-supergravity potential given in [14. More precisely, we consider the complete bosonic part of the action for a threedimensional supergravity with gauged PQ symmetries, in a form that is related to the one derived in [14] by a redualization of the PQ scalars to vectors. The redualized form is well suited for the comparison with the theories from fourfold compactifications with fluxes.

The potential was used in [2] to show that a flux proportional either to the holomorphic 4-form $\Omega$ (and its complex conjugate) or the square of the Kähler form $J$ of the Calabi-Yau classically leads to a vanishing cosmological constant, despite the complete (spontaneous) breaking of supersymmetry. This feature had been noticed before in [12] (for $F_{4} \sim \Omega+$ c.c.) and [19] (for $F_{4} \sim J \wedge J$ ). Here we further investigate the issue of supersymmetry breaking, and find that it is possible to obtain partial breaking from $N=2$ to $N=1$ by turning on a certain combination of both of these fluxes, i.e. $F_{4}=F J \wedge J+(\tilde{F} \Omega+$ c.c. $)$. These threedimensional $N=1$ vacua might be interesting due to Witten's proposal to explain the vanishing of the cosmological constant by considering our four-dimensional world to be described by the strong coupling limit of an $N=1$ supersymmetric three-dimensional theory [20].

The article is organized as follows. In section 2 we start with a summary of relevant facts about three-dimensional gauged supergravity with $N=2$, i.e. four supercharges) [14]. This theory is formulated with all vectors dualized to scalars, such that the bosonic spectrum contains only scalars from chiral multiplets as dynamical degrees of freedom. In order to make contact with the effective theory from Calabi-Yau fourfold compactifications, we then specialize to the case that some of the scalars have a PQ symmetry, which is the symmetry that is gauged. For this special case we perform a (re-)dualization of the PQ scalars to vectors and derive the general form of the bosonic part of the effective action. Furthermore, we discuss the condition for unbroken $N=2$ and $N=1$ supersymmetry. In section 3 we compare the general form of the gauged supergravities discussed in 
section 2 to those from Calabi-Yau fourfold compactifications of eleven-dimensional supergravity. We start by reviewing the case without 4-form flux, discussed before in 21]. As opposed to 21] however, here we express the effective action through both chiral and vector multiplets to make contact with the results in section 2 . The reason is that it is in these coordinates that the flux-induced potential can be expressed most easily. This is done in the following subsection, where we turn on 4 -form flux. We generalize the analysis of [18] by including the moduli from expanding the 3 -form in a basis of 3 -forms on the Calabi-Yau, finding that the potential is not modified in this case. Furthermore, we make some observations about the range of validity of our derivation. We end section 3 with a discussion of partial supersymmetry breaking to $N=1$, specializing the general condition from section 2 to the case at hand. By giving a concrete (toy) example we show that it is indeed possible to fulfill this condition. Finally, an appendix contains some more details on the search for an example of partial supersymmetry breaking.

\section{The gauged supergravity}

In this section, we first review the general form of $N=2$ gauged supergravity in three dimensions as given in [14, generalizing the abelian $U(1)$ gaugings of 22 , 23. We then turn to gauge groups that are exclusively generated by translational (Peccei-Quinn) symmetries of the scalar target space and show that these theories admit a dual formulation in which the PQ scalars are redualized into vector fields. From the supersymmetry transformation rules, we eventually derive the conditions of $N=1,2$ preserved supersymmetries at a stationary point of the scalar potential.

\section{$2.1 \quad d=3, N=2$ supergravity}

We start from the formulation of ungauged $d=3, N=2$ supergravity in which all vector fields are dualized into scalars. The total scalar target space is a Kähler manifold which we parametrize by complex coordinates $\left\{\phi^{i}, \bar{\phi}^{\bar{\imath}}\right\}$ with the metric derived from the Kähler potential $g_{i \bar{\imath}}=\partial_{i} \partial_{\bar{\imath}} K$. An isometry is described by a holomorphic vector field $\partial_{\bar{\imath}} X^{j}=0$, satisfying

$$
\nabla_{i} X_{\bar{\jmath}}+\nabla_{\bar{\jmath}} X_{i}=0
$$

Gauging a subgroup of isometries that is generated by a basis $\left\{X_{A}^{j}\right\}$ corresponds to defining space-time vector fields $A_{A \mu}$ via the duality equation

$$
\epsilon_{\mu \nu \rho} F_{A}^{\nu \rho}=-4 \Re\left[g_{i \bar{\imath}} X_{A}^{\bar{\imath}} D_{\mu} \phi^{i}\right]+\text { fermions },
$$

\footnotetext{
${ }^{6}$ We take the signature of the space-time metric to be $(+--)$. The $\epsilon_{\mu \nu \rho}$ is defined to transform as a tensor, i.e. $\epsilon_{123}=e, \epsilon^{123}=e^{-1}$. Our curvature conventions are $R_{\mu \nu \sigma}{ }^{\tau}=$ $\partial_{\nu} \Gamma_{\mu \sigma}^{\tau}+\Gamma_{\nu \lambda}^{\tau} \Gamma_{\mu \sigma}^{\lambda}-(\mu \leftrightarrow \nu), R_{\mu \nu}=R_{\mu \tau \nu}^{\tau}$.
} 
and minimally coupling them to the scalar fields by means of a constant symmetric matrix $\Theta^{A B}$ as

$$
D_{\mu} \phi^{j}=\partial_{\mu} \phi^{i}+g \Theta^{A B} A_{A \mu}\left(X_{B}^{i} \partial_{i}+X_{B}^{\bar{\imath}} \partial_{\bar{\imath}}\right) \phi^{j} .
$$

The duality equation (2.2) consistently implies the scalar equation of motion as integrability condition. Supersymmetry further requires the introduction of fermionic mass terms, a Chern-Simons term for the vector fields that gives rise to (2.2) as field equation, and a scalar potential. The gravitino mass tensor is given by

$$
A_{1}=\left(\begin{array}{rr}
2 T & 0 \\
0 & 2 T
\end{array}\right)+e^{K / 2}\left(\begin{array}{rr}
-\Re W & \Im W \\
\Im W & \Re W
\end{array}\right),
$$

with $T \equiv \mathcal{P}_{A} \Theta^{A B} \mathcal{P}_{B}$, where the real function $\mathcal{P}_{A}$ is the momentum map associated with the isometry $X_{A}^{i}$

$$
\partial_{j} \mathcal{P}_{A} \equiv \frac{1}{2} \mathrm{i} g_{j \bar{\jmath}} X_{A}^{\bar{j}}
$$

The gauging is uniquely determined by the choice of $\Theta^{A B}$ up to the superpotential $W$ which is an arbitrary holomorphic function of the scalar fields, subject to

$$
X_{A}^{i} D_{i} W=2 \mathrm{i} W \mathcal{P}_{A}
$$

with the Kähler covariant derivative $D_{i} W \equiv \partial_{i} W+\partial_{i} K W$. The complete bosonic part of the Lagrangian is given by

$$
e^{-1} \mathcal{L}_{g}=\frac{1}{2} R-g_{i \bar{\jmath}} D_{\mu} \phi^{i} D^{\mu} \bar{\phi}^{\bar{\jmath}}-\frac{1}{4} g \Theta^{A B} \epsilon_{\mu \nu \rho} A_{A}^{\mu} F_{B}^{\nu \rho}+V,
$$

with a scalar potential

$$
V=g^{2}\left(4 g^{i \bar{\imath}} \partial_{i} T \partial_{\bar{\imath}} T-4 T^{2}+e^{K}\left(\frac{1}{4} g^{i \bar{\imath}} D_{i} W D_{\bar{\imath}} \bar{W}-|W|^{2}\right)\right) .
$$

Summarizing, the gauged deformation of the $N=2$ supergravity is uniquely determined by the constant matrix $\Theta^{A B}$ describing the minimal coupling (2.3), and a holomorphic superpotential $W$ satisfying (2.6). It further induces fermionic mass terms as well as a Chern-Simons term and the scalar potential in the Lagrangian. Note that after gauging, the scalar fields may in general no longer be redualized into vector fields.

\subsection{Redualizing}

Let us now specialize this construction to gauge groups that are generated by translational symmetries of the scalar target space. As it turns out, in these theories the charged scalar fields may be redualized into vector fields and completely removed from the Lagrangian. Their removal turns the formerly nonpropagating vector fields into physical fields that appear with a Yang-Mills kinetic term and an 
additional Chern-Simons coupling in the Lagrangian. These are the theories that describe the compactifications of eleven-dimensional supergravity on Calabi-Yau fourfolds with background fluxes.

We introduce the following split of scalar fields

$$
\phi^{i}=\left(\phi^{a}, \phi^{A}\right),
$$

and define the real components $\varphi^{A}=\Re \phi^{A}, \hat{\varphi}^{A}=\Im \phi^{A}$. We now assume that the Kähler potential does not depend on the $\hat{\varphi}^{A}$

$$
K=K\left(\phi^{a}, \varphi^{A}\right) .
$$

In particular, this implies that

$$
g_{i \bar{\jmath}} \equiv\left(\begin{array}{ll}
g_{a \bar{b}} & g_{a B} \\
g_{A \bar{b}} & g_{A B}
\end{array}\right),
$$

with $g_{a \bar{b}}=\partial_{a} \partial_{\bar{b}} K, g_{a A}=\frac{1}{2} \partial_{a} \partial_{A} K$, and the real matrix $g_{A B}=\frac{1}{4} \partial_{A} \partial_{B} K \equiv \frac{1}{2} G_{A B}$. The partial derivatives $\partial_{A}$ here always refer to real derivatives w.r.t. the field $\varphi^{A}$ in (2.10). To avoid confusion, small $g$ will always refer to the metric obtained from the original Kähler potential $K$ in the completely dualized picture in which everything is expressed in chiral multiplets.

The geometry described by (2.10) is invariant under shifts in $\hat{\varphi}^{A}$, i.e. the metric (2.11) admits a set of "elementary" isometries, labelled by a subscript $B$, which are generated by the constant imaginary vector fields

$$
X_{B}^{i}=\left(0, \mathrm{i} \delta_{B}^{A}\right)
$$

in the basis (2.9). From (2.5) we find that the associated momentum map is given by

$$
\mathcal{P}_{A}=\frac{1}{4} \partial_{A} K
$$

The most general gauging of these shift isometries is described by a constant matrix $\Theta^{A B}$ such that the covariant derivatives become

$$
D_{\mu} \hat{\varphi}^{A}=\partial_{\mu} \hat{\varphi}^{A}+g \Theta^{A B} A_{B \mu},
$$

where the vector field is defined by the duality equation (2.2)

$$
F_{A \mu \nu}=-\epsilon_{\mu \nu \rho}\left(G_{A B}\left(\partial^{\rho} \hat{\varphi}^{B}+g \Theta^{B C} A_{C}^{\rho}\right)+2 \Im\left[g_{a A} \partial^{\rho} \phi^{a}\right]\right)
$$

Let us assume that the submatrix $G_{A B}$ of $g_{i \bar{\imath}}$ is separately invertible with inverse $G^{A B}$. Then $\partial^{\rho} \hat{\varphi}^{B}$ may be expressed in terms of $F_{A \mu \nu}$, and we derive that

$$
D^{\mu}\left(G^{A B} F_{B \mu \nu}\right)=\frac{1}{2} g \Theta^{A B} \epsilon_{\nu \mu \rho} F_{B}^{\mu \rho}+2 \epsilon_{\nu \mu \rho} \Im\left[\partial^{\mu}\left(G^{A B} g_{a B}\right) \partial^{\rho} \phi^{a}\right] .
$$


That is, the scalar fields $\hat{\varphi}^{A}$ can be eliminated from all equations of motion. In turn, the vector fields now satisfy a second order field equation which may be derived from the Lagrangian

$$
\frac{1}{4} G^{A B} F_{A}^{\mu \nu} F_{B \mu \nu}+\frac{1}{4} g \Theta^{A B} \epsilon_{\mu \nu \rho} A_{A}^{\mu} F_{B}^{\nu \rho}+\epsilon_{\mu \nu \rho} F_{A}^{\mu \nu} G^{A B} \Im\left[g_{a B} \partial^{\rho} \phi^{a}\right] .
$$

The remaining kinetic terms of the Lagrangian after redualization are given by

$$
\begin{aligned}
-\frac{1}{2} G_{A B} \partial_{\mu} \varphi^{A} \partial^{\mu} \varphi^{B}- & 2 \Re\left[g_{a A} \partial_{\mu} \varphi^{A} \partial^{\mu} \phi^{a}\right]-\left(g_{a \bar{b}}-g_{a A} G^{A B} g_{\bar{b} B}\right) \partial_{\mu} \phi^{a} \partial^{\mu} \bar{\phi}^{\bar{b}} \\
& -\frac{1}{2} g_{a A} G^{A B} g_{b B} \partial_{\mu} \phi^{a} \partial^{\mu} \phi^{b}-\frac{1}{2} g_{\bar{b} A} G^{A B} g_{\bar{b} B} \partial_{\mu} \bar{\phi}^{\bar{b}} \partial^{\mu} \bar{\phi}^{\bar{b}} .
\end{aligned}
$$

Defining new real coordinates

$$
M_{A} \equiv \frac{1}{2} \partial_{A} K
$$

such that $\partial_{A} M_{B}=G_{A B}, \partial_{a} M_{A}=g_{a A}$, the complete Lagrangian after redualization then takes the form

$$
\begin{aligned}
e^{-1} \mathcal{L}_{g}= & \frac{1}{2} R-\frac{1}{2} G^{A B} \partial_{\mu} M_{A} \partial^{\mu} M_{B}+\frac{1}{4} G^{A B} F_{A}^{\mu \nu} F_{B \mu \nu}-G_{a \bar{b}} \partial_{\mu} \phi^{a} \partial^{\mu} \bar{\phi}^{\bar{b}} \\
& +\epsilon_{\mu \nu \rho} F_{A}^{\mu \nu} \Im\left[G^{A B} g_{a B} \partial^{\rho} \phi^{a}\right]+\frac{1}{4} g \Theta^{A B} \epsilon_{\mu \nu \rho} A_{A}^{\mu} F_{B}^{\nu \rho}+V
\end{aligned}
$$

with the scalar potential $V$ from (2.8) above and the metric

$$
G_{a \bar{b}} \equiv\left(G^{a \bar{b}}\right)^{-1} \equiv\left(g^{a \bar{b}}\right)^{-1}=g_{a \bar{b}}-2 g_{a A} G^{A B} g_{\bar{b} B}
$$

To clarify the last equation, we note that the inverse of $g_{i \bar{\jmath}}(2.11)$ is given by

$$
g^{i \bar{\jmath}}=\left(\begin{array}{cc}
g^{a \bar{b}} & -2 g^{a \bar{b}} g_{\bar{b} B} G^{B A} \\
-2 g^{a \bar{b}} g_{\bar{b} B} G^{B A} & 2 G^{A B}+4 G^{A C} g_{a C} g^{a \bar{b}} g_{\bar{b} D} G^{D B}
\end{array}\right),
$$

where $g^{a \bar{b}}=\left(g_{a \bar{b}}-2 g_{a A} G^{A B} g_{\bar{b} B}\right)^{-1}$. Hence, the metrics which appear in the kinetic terms of the dualized Lagrangian (2.16) are not part of the blocks of the original Kähler metric (2.11) or its inverse (2.17), but are instead given by

$$
G_{A B}=2 g_{A B}, \quad G^{A B} \neq \frac{1}{2} g^{A B}, \quad G_{a \bar{b}} \neq g_{a \bar{b}}, \quad G^{a \bar{b}}=g^{a \bar{b}} .
$$

We should emphasize that these metrics as well as the mixed coupling in the dualized Lagrangian (2.16) can all be expressed via a "kinetic potential" $\tilde{K}\left(\phi^{\alpha}, M_{A}\right)$ as

$$
G^{A B}=-\frac{1}{2} \partial_{M_{A}} \partial_{M_{B}} \tilde{K}, \quad G_{a \bar{b}}=\partial_{\phi^{a}} \partial_{\phi^{\bar{b}}} \tilde{K}, \quad G^{A B} g_{a B}=\frac{1}{2} \partial_{\phi^{a}} \partial_{M_{A}} \tilde{K},
$$

where $\tilde{K}$ is given by

$$
\tilde{K}=K-2 M_{A} \varphi^{A}
$$


Moreover, the original Kähler potential $K$ can be recovered from $\tilde{K}$ as

$$
K=\tilde{K}-M_{A} \partial_{M_{A}} \tilde{K} .
$$

The scalar potential $V$ in (2.16) finally is expressed in terms of $T$ and a holomorphic function $W$. From (2.13) we find that the consistency constraint (2.6) is simply equivalent to assuming that $W=W\left(\phi^{\alpha}\right)$ is a holomorphic function of the fields $\phi^{\alpha}$ only. In the new coordinates $\left(\phi^{a}, M_{A}\right)$, the potential (2.8) simplifies to

$$
\begin{aligned}
g^{-2} V= & \frac{1}{2} M_{A} \Theta^{A C} G_{C D} \Theta^{D B} M_{B}-\frac{1}{4}\left(M_{A} \Theta^{A B} M_{B}\right)^{2} \\
& +\frac{1}{4} e^{K} G^{a \bar{b}} D_{a} W D_{\bar{b}} \bar{W}-e^{K}\left(1-\frac{1}{2} M_{A} G^{A B} M_{B}\right)|W|^{2},
\end{aligned}
$$

with the original Kähler potential $K$, a holomorphic function $W\left(\phi^{\alpha}\right)$ and the Kähler covariant derivatives $D_{a} W=\partial_{a} W+\left(\partial_{a} K\right) W$. Note that the metrics $G^{a \bar{b}}, G_{A B}$ appearing here are precisely the inverses of the metrics in the kinetic terms in (2.16), and that all mixed terms vanish. Furthermore, the potential can be expressed entirely in terms of the kinetic potential (2.19), using (2.18) and (2.20). Let us finally note that for $M_{A} G^{A B} M_{B}=2$, the last term in (2.21) vanishes, and the potential is in fact positive definite

$$
\begin{aligned}
g^{-2} V= & \frac{1}{2}\left(M_{A} \Theta^{A C}-2 T M_{A} G^{A C}\right) G_{C D}\left(\Theta^{D B} M_{B}-2 T G^{D B} M_{B}\right) \\
& +\frac{1}{4} e^{K} G^{a \bar{b}} D_{a} W D_{\bar{b}} \bar{W} .
\end{aligned}
$$

Summarizing, we have seen that in the special case of a gauging of the PecceiQuinn symmetries associated with some of the scalar fields, these fields may be dualized into vector fields. In the dual picture the physical fields are the complex scalars $\phi^{a}$, the real scalars $M_{A}$, and the vector fields $A_{A \mu}$; their dynamics is described by the Lagrangian (2.16) with scalar potential (2.21). This may be viewed as a deformation of the Lagrangian $\mathcal{L}_{g=0}$ that is triggered by the Chern-Simons term for the vector fields (which encodes the constant matrix $\Theta^{A B}$ ) whose supersymmetrization then induces the fermionic mass terms and the scalar potential. Again, this deformation is unique up to the choice of the holomorphic superpotential $W$.

A noteworthy property of the dualized Lagrangian is the appearance of the kinetic potential $\tilde{K}$ in the kinetic terms of (2.16), whereas the part of the potential (2.21) derived from a superpotential still involves the old Kähler potential $K$, both via the overall factor $e^{K}$ and the Kähler covariant derivatives. Let us remind the reader that a similar situation occurs in $D=4$ in an ungauged theory with both chiral and linear multiplets. (The latter become vector multiplets in a reduction to three dimensions and therefore the similarity is no accident.) Namely, consider the $D=4$ theory described by a Kähler potential [24, 25]

$$
K\left(\phi^{a}, \bar{\phi}^{\bar{a}}, L\right)=K_{0}\left(\phi^{a}, \bar{\phi}^{\bar{a}}\right)+\alpha \ln L,
$$


where $\phi^{a}$ denote the (complex) scalars from the chiral multiplets, $L$ is one (real) scalar from the linear multiplet, and $\alpha$ is an arbitrary constant. The motivation to look at this special form of the Kähler potential in $D=4$ comes from the fact that it is exactly what one needs to describe the linear dilaton multiplet in heterotic string models. The effective action of the linear multiplet coupled to chiral multiplets involves another real function of the $\phi^{a}$, the 'linear potential' $V\left(\phi^{a}, \bar{\phi}^{\bar{a}}\right)$. In the context of the heterotic string this encodes the (non-holomorphic) threshold corrections to the gauge coupling and in the present context it is the analog of $\varphi$ in (2.19). More precisely, defining the kinetic potential

$$
\tilde{K}=K-3 L V,
$$

the bosonic part of the Lagrangian including a potential derived from a superpotential takes the form [24]

$$
\begin{aligned}
e^{-1} \mathcal{L}= & \frac{1}{2} R-\tilde{K}_{a \bar{a}} \partial_{\mu} \phi^{a} \partial^{\mu} \bar{\phi}^{\bar{a}}+\frac{1}{4} \tilde{K}_{L L} \partial_{\mu} L \partial^{\mu} L \\
& -\frac{1}{3 !} \tilde{K}_{L L} H_{\mu \nu \rho} H^{\mu \nu \rho}+\frac{1}{3 !} \epsilon_{\mu \nu \rho \sigma} H^{\nu \rho \sigma} \Im\left(\tilde{K}_{L a} \partial^{\mu} \phi^{a}\right) \\
& +e^{K}\left[\tilde{K}^{a \bar{a}} D_{a} W D_{\bar{a}} \bar{W}-\left(3-L K_{L}\right)|W|^{2}\right]
\end{aligned}
$$

where the covariant derivatives $D_{a} W=\left(\partial_{a}+K_{a}\right) W$ involve the old Kähler potential.8 Taking into account that (2.20) implies

$$
M_{A} M_{B}\left(\partial_{M_{A}} \partial_{M_{B}} \tilde{K}\right)=-M_{A}\left(\partial_{M_{A}} K\right),
$$

the similarity between (2.25) and (2.16), (2.21) is obvious, up to the terms depending on $\Theta^{A B}$ which correspond to the gauging in three dimensions.

\subsection{Supersymmetry breaking}

The fermionic part of the Lagrangian (2.16) and supersymmetry transformation rules may be extracted from [14] upon eliminating $\hat{\varphi}$ by means of (2.14). Here, we restrict to giving the supersymmetry variation of the gravitino, which is sufficient to determine the conditions of $N=1,2$ preserved supersymmetries at a stationary point of the scalar potential. From [14, we infer that

$$
\delta \psi_{\mu}^{I}=\nabla_{\mu} \epsilon^{I}+\mathrm{i} g A_{1}^{I J} \gamma_{\mu} \epsilon^{J}+\left(\frac{1}{2} \Re\left[\partial_{\mu} \phi^{i} \partial_{i} K\right]+g \Theta^{A B} A_{A \mu} \mathcal{P}_{B}\right) \varepsilon^{I J} \epsilon^{J},
$$

where the index $I=1,2$ labels the supercharges and $\varepsilon^{I J}$ is the antisymmetric tensor $\varepsilon^{12}=1$. The Killing spinor associated with a supersymmetric ground state of

\footnotetext{
${ }^{7}$ We use the term Kähler potential here although, like in the three-dimensional case, the scalar manifold is only Kähler when expressed in the dual, chiral variables.

${ }^{8}$ We have adopted the signs in the formula of 24] to our choice of conventions.
} 
the potential (2.21) (at which we may set $\partial_{\mu} \phi^{i}=0=A_{A \mu}$ ) is then given by tensoring a three-dimensional AdS/Minkowski Killing spinor with an eigenvector of $A_{1}$. Vanishing of (2.26) imposes the relation $g|\lambda|=\sqrt{-V_{0}}$ between the corresponding eigenvalue $\lambda$ of $A_{1}^{I J}$ and the value of the potential $V_{0}$ at the critical point. With the eigenvalues of (2.4) given by

$$
\lambda_{ \pm}=-\frac{1}{2} M_{A} \Theta^{A B} M_{B} \pm e^{K / 2}|W|,
$$

this condition translates into

$$
\begin{aligned}
\pm 2 e^{K / 2} M_{A} \Theta^{A B} M_{B}|W| \equiv & M_{A} \Theta^{A C} G_{C D} \Theta^{D B} M_{B}+e^{K} M_{A} G^{A B} M_{B}|W|^{2} \\
& +\frac{1}{2} e^{K} G^{a \bar{b}} D_{a} W D_{\bar{b}} \bar{W}
\end{aligned}
$$

In order to preserve the full $N=2$ supersymmetry, i.e. satisfy this equation for both $\lambda_{+}$and $\lambda_{-}$, both sides of this equation must vanish, thus implying the conditions

$$
\Theta^{A B} M_{B} \equiv 0, W \equiv 0, \quad D_{a} W \equiv 0 .
$$

This obviously also implies stationarity of the potential (2.21) as well as vanishing of the cosmological constant. For an extremum with $N=1$ supersymmetry, it remains to reconcile stationarity of (2.21) with the condition (2.27) for one choice of sign. As a special case, we may consider the positive definite potential (2.22), for which $V_{0}=0$ is a sufficient condition for a minimum. The latter is equivalent to

$$
\Theta^{D B} M_{B}-2 T G^{D B} M_{B}=0, \quad D_{a} W=0,
$$

while the condition (2.27) in this case reduces to

$$
M_{A} \Theta^{A B} M_{B}= \pm 2 e^{K / 2}|W| .
$$

The two conditions (2.29), 2.30) are necessary and sufficient for the existence of a minimum with $N=1$ supersymmetry and vanishing cosmological constant in the positive definite potential (2.22).

\section{$3 \quad$ Kaluza-Klein reduction}

In this section we want to make contact between the general discussion of the previous section and Calabi-Yau fourfold compactification of eleven-dimensional supergravity. We start by reviewing the case without flux and then consider the modifications in its presence. Without flux the reduction leads to an ungauged supergravity, whose scalar manifold has some PQ symmetries. These are then gauged by turning on the flux. 


\subsection{Compactification without flux}

The compactification without flux was investigated in [21. 9] The starting point is the eleven-dimensional supergravity with (bosonic) Lagrangian [26]

$$
\begin{aligned}
e^{-1} \mathcal{L}^{(11)}= & \frac{1}{2} R^{(11)}+\frac{1}{4 \cdot 4 !} F_{M_{1} \ldots M_{4}} F^{M_{1} \ldots M_{4}} \\
& +\frac{1}{12 \cdot 3 !(4 !)^{2}} A_{M_{1} M_{2} M_{3}} F_{M_{4} \ldots M_{7}} F_{M_{8} \ldots M_{11}} \epsilon^{M_{1} \ldots M_{11}}
\end{aligned}
$$

where we have adapted the signs to our conventions. The (bosonic) spectrum only consists of the metric and a 3 -form gauge field. The Lagrangian (3.1) is the leading order contribution in a derivative expansion. One of the next-to-leadingorder terms in this expansion reads [27, 28]

$$
e^{-1} \mathcal{L}_{1}^{(11)}=-\frac{T_{2}}{3 ! 8 !} A_{M_{1} \ldots M_{3}} X_{M_{4} \ldots M_{11}} \epsilon^{M_{1} \ldots M_{11}}
$$

with

$$
X_{8}=\frac{1}{(2 \pi)^{4}}\left(-\frac{1}{768}\left(\operatorname{tr} R^{2}\right)^{2}+\frac{1}{192} \operatorname{tr} R^{4}\right)
$$

and $T_{2} \equiv(2 \pi)^{2 / 3}\left(2 \kappa_{11}^{2}\right)^{-1 / 3}$ is the membrane tension. The term (3.2) leads to an important constraint for Calabi-Yau fourfold reductions of eleven-dimensional supergravity. Due to the relation

$$
\int_{Y_{4}} X_{8}=-\frac{\chi}{24}
$$

the term (3.2) potentially induces a tadpole-term for the three-form $A_{3}$ in compactifications on Calabi-Yau fourfolds with non-vanishing Euler number $\chi$, which would render the resulting vacuum inconsistent [29]. However, the coefficient of the tadpole term gets further contributions from space-time filling membranes, non-trivial $F_{4}$-flux [15, 29, 30] or M5-branes wrapped around three-cycles in the Calabi-Yau [16]. As we do not consider any space-time filling membranes or wrapped M5branes here, the relation

$$
\frac{1}{4 \kappa_{11}^{2}} \int_{Y_{4}} F_{4} \wedge F_{4}=\frac{T_{2}}{24} \chi
$$

has to hold for consistency. Postponing the discussion of non-trivial flux to the next section, we have to consider fourfolds with $\chi=0$ for the moment.

\footnotetext{
${ }^{9}$ Here we extend however the results of [21] in that we make contact to the formulas derived in the last section, i.e. we formulate the theory using both, chiral and vector multiplets.

${ }^{10}$ We use the subscript $\mathcal{L}_{1}^{(11)}$ here, because there will be further higher derivative terms relevant in the next section.
} 
Let us now discuss the three-dimensional bosonic spectrum. Compactifying on a Calabi-Yau fourfold, from the metric one gets a graviton, $h^{1,1}\left(Y_{4}\right)$ Kähler moduli and $h^{3,1}\left(Y_{4}\right)$ complex structure moduli $Z^{\alpha}$. As we are interested in discussing the large volume expansion later on it is convenient to use rescaled Kähler moduli making the dependence on the constant background volume explicit. Thus the volume of the Calabi-Yau is given by

$$
\operatorname{Vol}\left(Y_{4}\right)=\mathcal{V}_{0} \tilde{\mathcal{V}}\left(\tilde{M}_{A}\right)
$$

where $\mathcal{V}_{0}$ is the background volume and $\tilde{\mathcal{V}}$ depends on the rescaled Kähler moduli $\tilde{M}_{A}$. Furthermore, one expands the 3 -form

$$
A_{\mu i \bar{\jmath}}=\sum_{A=1}^{h^{1,1}} \mathcal{V}_{0}^{1 / 4} A_{A \mu} e_{i \bar{\jmath}}^{A} \quad, \quad A_{i j \bar{k}}=\sum_{I=1}^{h^{2,1}} \mathcal{V}_{0}^{3 / 8} N_{I} \Psi_{i j \bar{k}}^{I}
$$

where we have introduced bases $e^{A}$ for $H^{1,1}\left(Y_{4}\right)$ and $\Psi^{I}$ for $H^{2,1}\left(Y_{4}\right)$. Thus the spectrum comprises $h^{1,1}\left(Y_{4}\right)$ vector multiplets and $h^{2,1}\left(Y_{4}\right)+h^{3,1}\left(Y_{4}\right)$ chiral multiplets. The explicit factors of $\mathcal{V}_{0}$ have been introduced in (3.7) because only for fields so defined, the kinetic terms stemming from the reduction of the eleven-dimensional supergravity action are of the same order in the background volume for all the scalars and vectors.

In order to derive the low energy effective action one has to take into account that the Hodge decomposition $H^{3}\left(Y_{4}\right)=H^{1,2}\left(Y_{4}\right)+H^{2,1}\left(Y_{4}\right)$ depends on the complex structure. The basis $\Psi^{I}$ of $(2,1)$-forms can locally be chosen to depend holomorphically on the complex structure or, in other words,

$$
\bar{\partial}_{\bar{Z}^{\bar{\alpha}}} \Psi^{I}=0, \quad \partial_{Z^{\alpha}} \Psi^{I} \neq 0 .
$$

The derivative $\partial_{Z^{\alpha}} \Psi^{I}$ can be expanded into $(1,2)$ - and $(2,1)$-forms with complexstructure dependent coefficient functions $\sigma$ and $\tau$

$$
\partial_{Z^{\alpha}} \Psi^{I}=\sigma_{\alpha K}^{I}(Z, \bar{Z}) \Psi^{K}+\tau_{\alpha \bar{L}}^{I}(Z, \bar{Z}) \bar{\Psi}^{\bar{L}}
$$

Using this when inserting (3.7) and the usual product ansatz for the metric into (3.1), the three-dimensional effective Lagrangian takes the form

$$
\begin{aligned}
e^{-1} \mathcal{L}_{0}= & \frac{1}{2} R-G_{\alpha \bar{\beta}} \partial_{\mu} Z^{\alpha} \partial^{\mu} \bar{Z}^{\bar{\beta}}-\frac{1}{2} G^{A B} \partial_{\mu} M_{A} \partial^{\mu} M_{B} \\
& +\frac{1}{4} G^{A B} F_{A}^{\mu \nu} F_{B \mu \nu}-G^{I \bar{J}} D_{\mu} N_{I} D^{\mu} \bar{N}_{\bar{J}} \\
& -\frac{1}{8} \epsilon^{\mu \nu \rho} d^{A I \bar{J}} F_{A \mu \nu}\left[N_{I} D_{\rho} \bar{N}_{\bar{J}}-D_{\rho} N_{I} \bar{N}_{\bar{J}}\right] .
\end{aligned}
$$

\footnotetext{
${ }^{11}$ Here we performed a Weyl-rescaling $g_{\mu \nu} \rightarrow \tilde{\mathcal{V}}^{2} g_{\mu \nu}$ and a further rescaling of the Kähler moduli similar to the one in 18 , i.e. $M_{A}=\tilde{M}_{A} \tilde{\mathcal{V}}^{-1}$.
} 
The corresponding action is given by $\kappa_{3}^{-2} \int d^{3} x \mathcal{L}_{0}$ and the three-dimensional gravitational constant is related to the eleven-dimensional one by $\kappa_{3}^{-2}=\kappa_{11}^{-2}\left(\mathcal{V}_{0} \kappa_{11}^{16 / 9}\right)$. Let us explain the notation in (3.10). . $^{2}$ The scalar sigma-model is described by the three metrics $G_{\alpha \bar{\beta}}, G^{A B}$ and $G^{I J}$. The Zamolodchikov metric $G_{\alpha \bar{\beta}}$ on the complex structure moduli space is Kähler with Kähler potential

$$
K_{\mathrm{CS}}=-\ln \left(\int_{Y_{4}} \Omega \wedge \bar{\Omega}\right)
$$

where $\Omega$ is the $(4,0)$-form of $Y_{4}$. Similarly, the metric on the Kähler moduli space is given by the second derivative of a real function

$$
G^{A B}=-\frac{1}{2} \partial_{M_{A}} \partial_{M_{B}} K_{\mathrm{K}}=-\frac{1}{2} \partial_{M_{A}} \partial_{M_{B}} \ln \mathcal{V},
$$

where $\mathcal{V}=\frac{1}{4 !} \int_{Y_{4}} J^{4}=\frac{1}{4 !} d^{A B C D} M_{A} M_{B} M_{C} M_{D}$ is a rescaled volume of $Y_{4}, J=M_{A} e^{A}$ being a rescaled Kähler form and

$$
d^{A B C D}=\int_{Y_{4}} e^{A} \wedge e^{B} \wedge e^{C} \wedge e^{D}
$$

the quadruple intersection numbers. Also

$$
G^{I \bar{J}}=-\frac{i}{2} d^{A I \bar{J}} M_{A}
$$

where we have used the definition

$$
d^{A I \bar{J}} \equiv \int_{Y_{4}} e^{A} \wedge \Psi^{I} \wedge \bar{\Psi}^{\bar{J}}
$$

Furthermore, we introduced the abbreviations

$$
D_{\mu} N_{I}=\partial_{\mu} N_{I}+N_{K} \sigma_{\alpha I}^{K} \partial_{\mu} Z^{\alpha}+\bar{N}_{\bar{L}} \bar{\tau}_{\bar{\beta} I}^{\bar{L}} \partial_{\mu} \bar{Z}^{\bar{\beta}}, \quad D_{\mu} \bar{N}_{\bar{J}}=\overline{D_{\mu} N_{J}}
$$

Let us next make contact with the formulas of the previous chapter. To do so we have to express (3.10) in the adequate variables, i.e. the chiral fields should be those appearing also in the dualized version without vector multiplets, and the scalars from the vector multiplets should obey (2.15). The correct Kähler coordinates in the dualized theory were determined in [21]. In the case without flux, the dualization can be performed explicitly by adding a Lagrange multiplier term

$$
e^{-1} \mathcal{L}_{\mathrm{L} . \mathrm{M} .}=F_{\mu}^{A} \partial^{\mu} P_{A} \quad, \quad F^{A \rho}=\frac{1}{2} \epsilon^{\rho \mu \nu} F_{\mu \nu}^{A}
$$

\footnotetext{
${ }^{12}$ Note that the notation slightly differs from [21] in order to be consistent with the notation of section 2 .
} 
and eliminating the fields $F_{\mu}^{A}$ via their equations of motion. The Kähler structure of the resulting sigma-model becomes manifest when expressed in the coordinates $\phi^{i}=\left(Z^{\alpha}, \hat{N}^{I}, \phi^{A}\right)$, where

$$
\begin{aligned}
& \hat{N}^{I}=\hat{G}^{I \bar{J}}\left(Z^{\alpha}, \bar{Z}^{\bar{\alpha}}\right) \bar{N}_{\bar{J}}, \\
& \phi^{A}=i P^{A}-2 \mathcal{V}^{A} \mathcal{V}^{-1}+\frac{i}{4} d^{A M \bar{L}} \hat{G}_{\bar{J} M} \hat{G}_{\bar{L} I} \hat{N}^{I} \overline{\hat{N}}^{\bar{J}}-\omega_{I K}^{A} \hat{N}^{I} \hat{N}^{K} .
\end{aligned}
$$

Here we have defined a variant of (3.14) which is independent of the Kähler moduli,

$$
\hat{G}^{I \bar{J}}=-\frac{i}{2} d^{A I \bar{J}} c_{A}
$$

where all $c_{A}$ can be chosen to be equal to 1 , and

$$
\mathcal{V}^{A} \equiv \frac{1}{4 !} \int_{Y_{4}} e^{A} \wedge J \wedge J \wedge J
$$

Furthermore, the $\omega_{I K}^{A}$ are functions of $Z^{\alpha}$ and $\bar{Z}^{\bar{\alpha}}$ which have to obey

$$
\bar{\partial}_{\bar{Z} \bar{\alpha} \omega_{I K}}^{A}=-\frac{i}{4} \hat{G}_{\bar{L} I} \hat{G}_{\bar{J} K} d^{A M \bar{L}_{\bar{\tau}} \overline{\bar{J}}}{ }_{\bar{\alpha} M}
$$

but are otherwise unconstrained. In terms of these coordinates the Kähler potential is given by

$$
K=K_{\mathrm{CS}}+K_{\mathrm{K}}
$$

where $K_{\mathrm{CS}}$ and $K_{\mathrm{K}}$ are given in (3.11) and (3.12) and have to be expressed in terms of the Kähler coordinates $\phi^{i}$. Doing so one realizes that $K$ indeed does not depend on the $\Im\left(\phi^{A}\right)$.

Now one can show that

$$
\partial_{\phi^{A}} K=M_{A}
$$

which indeed coincides with the general formula (2.15).13 Hence the appropriate variables for making contact with the last chapter are $\phi^{a}=\left(Z^{\alpha}, \hat{N}^{I}\right)$ and $\left(M_{A}, A_{A \mu}\right)$. In the new coordinates the 'covariant' derivatives (3.16) become

$$
\begin{aligned}
& D_{\mu} \bar{N}_{\bar{J}}=\hat{G}_{\bar{J} I} \partial_{\mu} \hat{N}^{I}+\partial_{\mu} Z^{\alpha}\left(\hat{G}_{\bar{M} K} \overline{\hat{N}}^{\bar{M}} \tau_{\alpha \bar{J}}^{K}-\hat{G}_{\bar{J} I} \hat{N}^{K} \sigma_{\alpha K}^{I}\right), \\
& D_{\mu} N_{I}=\hat{G}_{\bar{J} I} \partial_{\mu} \overline{\hat{N}}^{\bar{J}}+\partial_{\mu} \bar{Z}^{\bar{\beta}}\left(\hat{G}_{\bar{M} K} \hat{N}^{K} \bar{\tau}_{\bar{\beta} I}^{\bar{M}}-\hat{G}_{\bar{J} I} \overline{\hat{N}}^{\bar{M}} \bar{\sigma}_{\bar{\beta} \bar{M}}^{\bar{J}}\right),
\end{aligned}
$$

as can be seen from

$$
\partial_{Z^{\alpha}} \hat{G}^{I \bar{J}}=\sigma_{\alpha K}^{I} \hat{G}^{K \bar{J}}, \quad \partial_{Z^{\alpha}} d^{A I \bar{J}}=\sigma_{\alpha K}^{I} d^{A K \bar{J}} .
$$

\footnotetext{
${ }^{13}$ Notice that $K$ in (3.23) only depends on the real part $\varphi^{A}=\Re \phi^{A}$. Thus $\partial_{\phi^{A}} K=\frac{1}{2} \partial_{\varphi^{A}} K$.
} 
Plugging them into (3.10) one can read off $G_{a \bar{b}}$ (which will be given below, in (3.38)). Moreover, from (2.19) and (3.19) we see that the kinetic potential $\tilde{K}$ is given by

$$
\tilde{K}=K-M_{A}\left(\frac{i}{2} d^{A M \bar{L}} \hat{G}_{\bar{J} M} \hat{G}_{\bar{L} I} \hat{N}^{I} \overline{\hat{N}}^{\bar{J}}-\omega_{I K}^{A} \hat{N}^{I} \hat{N}^{K}-\bar{\omega}_{\bar{J} \bar{L}}^{A} \overline{\hat{N}}^{\bar{J}} \overline{\hat{N}}^{\bar{L}}\right) .
$$

Indeed, one can show

$$
G^{A B}=-\frac{1}{2} \partial_{M_{A}} \partial_{M_{B}} \tilde{K}, \quad G_{a \bar{b}}=\partial_{\phi^{a}} \partial_{\bar{\phi}^{\bar{b}}} \tilde{K}
$$

and

$$
d^{A I \bar{J}}\left[N_{I} D_{\rho} \bar{N}_{\bar{J}}-D_{\rho} N_{I} \bar{N}_{\bar{J}}\right]=-4 \Im\left[\left(\partial_{M_{A}} \partial_{\phi^{a}} \tilde{K}\right) \partial_{\rho} \phi^{a}\right]+\partial_{\rho}(\ldots)
$$

where, again, first one has to express the left hand side through the actual Kähler coordinates (3.18). Using these formulas in (3.10) shows that the low energy effective Lagrangian $\mathcal{L}_{0}$ is indeed of the form (2.16), taking into account (2.18).

Let us finally mention that it is straightforward but rather tedious to verify the relations (2.17) and (2.14), in the ungauged case.

\subsection{Inclusion of 4 -form flux}

In this section we consider the effects of turning on a background flux for the 4form $F_{4}$. The case $h^{2,1}\left(Y_{4}\right)=0$ was already discussed in [18] and here we show that the same result (and calculation) still holds in the general case including the $(2,1)$-moduli $\hat{N}^{I}$.

Let us remind the reader that in order to calculate the potential in a CalabiYau fourfold compactification with 4-form fluxes one has to take into account also one of the higher-derivative terms of the eleven-dimensional theory. Only some of them are known explicitly at the moment of writing. ${ }^{10}$ There is a term

$$
e^{-1} \mathcal{L}_{2}^{(11)}=b_{1} T_{2}\left(J_{0}-\frac{1}{2} E_{8}\right)
$$

where $b_{1}^{-1} \equiv(2 \pi)^{4} 3^{2} 2^{13}$ and

$$
\begin{aligned}
& E_{8}=\frac{1}{3 !} \epsilon^{A B C M_{1} N_{1} \ldots M_{4} N_{4}} \epsilon_{A B C M_{1}^{\prime} N_{1}^{\prime} \ldots M_{4}^{\prime} N_{4}^{\prime}} R^{M_{1}^{\prime} N_{1}^{\prime}}{ }_{M_{1} N_{1}} \ldots R^{M_{4}^{\prime} N_{4}^{\prime}}{ }_{M_{4} N_{4}}, \\
& J_{0}=t^{M_{1} N_{1} \ldots M_{4} N_{4}} t_{M_{1}^{\prime} N_{1}^{\prime} \ldots M_{4}^{\prime} N_{4}^{\prime}} R^{M_{1}^{\prime} N_{1}^{\prime}}{ }_{M_{1} N_{1}} \ldots R^{M_{4}^{\prime} N_{4}^{\prime}}{ }_{M_{4} N_{4}}+\frac{1}{4} E_{8} .
\end{aligned}
$$

\footnotetext{
${ }^{14}$ This corresponds to (2.19) up to an irrelevant constant that can be absorbed by a Kähler transformation of $K$. Moreover, it is very reminiscent to the Kähler potential of the effective two-dimensional theory that one gets from a Calabi-Yau fourfold compactification of type IIA [31.

${ }^{15}$ See [32] for an overview and for references. We will use the notation of [33] in the following.
} 
The tensor $t$ is defined by $t^{M_{1} \ldots M_{8}} A_{M_{1} M_{2}} \ldots A_{M_{7} M_{8}}=24 \operatorname{tr} A^{4}-6\left(\operatorname{tr} A^{2}\right)^{2}$ for antisymmetric tensors $A$, and $E_{8}$ is an eleven-dimensional generalization of the eightdimensional Euler density. More generally one can define [33]

$$
\begin{aligned}
E_{n}\left(M_{D}\right)= & \frac{1}{(D-n) !} \epsilon_{N_{1} \ldots N_{D-n} N_{D-n+1} \ldots N_{D}} \epsilon^{N_{1} \ldots N_{D-n} N_{D-n+1}^{\prime} \ldots N_{D}^{\prime}} \\
& R^{N_{D-n+1} N_{D-n+2}} N_{D-n+1}^{\prime} N_{D-n+2}^{\prime} \ldots R^{N_{D-1} N_{D}} N_{D-1}^{\prime} N_{D}^{\prime},
\end{aligned}
$$

where $D$ denotes the real dimension of the manifold. Then $E_{8}\left(Y_{4}\right)$ is proportional to the eight-dimensional Euler density, i.e.

$$
12 b_{1} \int_{Y_{4}} d^{8} y \sqrt{g} E_{8}\left(Y_{4}\right)=\chi .
$$

Thus in a Kaluza-Klein reduction on a Calabi-Yau fourfold the $E_{8}$-term in (3.30) contributes to the potential of the three-dimensional effective theory, as has first been noted in 34. On the other hand, the integral of $J_{0}$ over the Calabi-Yau vanishes to lowest order in $\kappa_{11}$, i.e. for the Ricci-flat metric [35].

In [18] it was shown that the contribution of the Euler-term combines with the one from the kinetic term of the 4-form to give the potential of the effective theory to lowest order in the large volume limit. Other possible contributions to the potential might come e.g. from (a yet unknown) $F_{4}^{2} R^{3}$-term. However, this would be suppressed in the large volume limit as compared to the contributions of $\left|F_{4}\right|^{2}$ and $E_{8}$. We will come back to a discussion of the limit we are considering at the end of this section.

Repeating the calculation of $\left[18\right.$ in the case with $h^{(2,1)} \neq 0$, we see that a background flux $F_{4}$ leads to the appearance of a potential and a Chern-Simons term that was first noted in [16]. This was to be expected also from the general discussion in chapter 2 , cf. (2.16). Thus (3.10) gets corrected by

$$
e^{-1} \mathcal{L}_{g}=e^{-1} \mathcal{L}_{0}+\frac{1}{2} g \epsilon^{\mu \nu \rho} \Theta^{A B} A_{A \mu} F_{B \nu \rho}+g^{2} V,
$$

where $g=\mathcal{V}_{0}^{-1 / 2}$. The potential is given by

$$
V=e^{K} G^{\alpha \bar{\beta}} D_{\alpha} W D_{\bar{\beta}} \bar{W}+8 G_{A B} \partial^{A} T \partial^{B} T-16 T^{2},
$$

where the Kähler potential of the gauged theory is the same as the one of the ungauged theory given in (3.23). Furthermore, $D_{\alpha}=\partial_{\alpha}+\left(\partial_{\alpha} K\right)$ and the superpotential $W$ resp. the function $T$ are defined as

$$
W=\int_{Y_{4}} \Omega \wedge F_{4} \quad, \quad T=\frac{1}{16} \int_{Y_{4}} J \wedge J \wedge F_{4} .
$$

Finally, the coefficients of the Chern-Simons term are given by

$$
\Theta^{A B}=2 \partial^{A} \partial^{B} T=\frac{1}{4} \int_{Y_{4}} e^{A} \wedge e^{B} \wedge F_{4} .
$$


As we already said the potential is exactly the same as the one calculated in 18 without the $(2,1)$-moduli, as it only gets contributions from the kinetic term of the 3-form and the higher derivative term (3.31). Moreover, the form of the potential (3.35) is perfectly consistent with the general formula (2.21). Obviously the first line of (2.21) corresponds the last two terms in (3.35). Furthermore, the last term of (2.21) vanishes, leading to a no-scale potential. Finally, to verify that the first term in the second line of (2.21) corresponds to the first term in (3.35), we notice that $D_{I} W=0$ and that the metric $G_{a \bar{b}}$ has the form

$$
G_{a \bar{b}}=\left(\begin{array}{cc}
G_{\alpha \bar{\beta}}+\mathcal{M}_{K \bar{L}} \mathcal{B}_{\alpha}^{K} \overline{\mathcal{B}} \overline{\bar{\beta}} & \mathcal{M}_{K \bar{J}} \mathcal{B}_{\alpha}^{K} \\
\mathcal{M}_{I \bar{L}} \overline{\mathcal{B}} \overline{\bar{\beta}} & \mathcal{M}_{I \bar{J}}
\end{array}\right)
$$

where we have defined

$$
\mathcal{M}_{I \bar{J}}=G^{K \bar{L}} \hat{G}_{\bar{L} I} \hat{G}_{\bar{J} K}, \quad \mathcal{B}_{\alpha}^{I}=\hat{G}_{\bar{M} K} \overline{\hat{N}}^{\bar{M}} \tau_{\alpha \bar{L}}^{K} \hat{G}^{I \bar{L}}-\sigma_{\alpha K}^{I} \hat{N}^{K}
$$

The inverse metric is thus given by

$$
G^{\bar{b} a}=\left(\begin{array}{cc}
G^{\bar{\beta} \alpha} & -G^{\bar{\beta} \gamma} \mathcal{B}_{\gamma}^{I} \\
-G^{\bar{\delta} \alpha} \overline{\mathcal{B}}_{\bar{\delta}}^{\bar{J}} & \mathcal{M}^{\bar{J} I}+G^{\bar{\delta} \gamma} \mathcal{B}_{\bar{\delta}}^{\bar{J}} \mathcal{B}_{\gamma}^{I}
\end{array}\right)
$$

As the $(\bar{\beta}, \alpha)$-subsector of $G^{\bar{b} a}$ is just given by $G^{\bar{\beta} \alpha}$, as in the case without the $(2,1)$-moduli $\hat{N}^{I}$, we see that indeed (3.35) is consistent with (2.21).

Let us now comment on the kind of expansion we are making, i.e. the range of validity of (3.34). First of all we are considering the large volume limit, i.e. $\mathcal{V}_{0} \gg 1$. However, we also have to restrict the choice of the fourfold, in order to end up with (3.34) as the effective theory. For the Chern-Simons term and the potential to remain at leading order in the large volume limit, we have to consider Calabi-Yau fourfolds with large Euler number $\chi$. From (3.5) it is clear that the flux is of the order of the square root of the Euler number, $F_{4} \sim \chi^{1 / 2}$. Thus for $\chi \sim \mathcal{V}_{0}$ the Chern-Simons term and the potential are of the same order as the terms in $\mathcal{L}_{0}$. A second condition on the Calabi-Yau comes from the fact that we want to neglect all other contributions from higher derivative terms that are not contained in (3.34). As was observed in [18, the term (3.30) leads to a correction to the three-dimensional Einstein-Hilbert term, due to the fact that

$$
E_{8}\left(M_{3} \times Y_{4}\right)=E_{8}\left(Y_{4}\right)+4 E_{2}\left(M_{3}\right) E_{6}\left(Y_{4}\right)
$$

where $E_{2}\left(M_{3}\right)=2 R$. More precisely, before the Weyl-rescaling the three-dimensional Einstein-Hilbert term gets corrected to

$$
\mathcal{S}_{\mathrm{EH}}=\frac{1}{2 \kappa_{3}^{2}} \int d^{3} x e\left(\tilde{\mathcal{V}}+a \mathcal{V}_{0}^{-3 / 4} \int_{Y_{4}} d^{8} z \sqrt{\tilde{g}} E_{6}\left(Y_{4}\right)\right) R,
$$

for some constant $a$. Here we have used the Kähler coordinates $\tilde{M}_{A}$ of (3.6) again, i.e. $\tilde{J}=\tilde{M}_{A} e^{A}$. It can be shown that

$$
\int_{Y_{4}} d^{8} z \sqrt{\tilde{g}} E_{6}\left(Y_{4}\right) \sim \int_{Y_{4}} \tilde{J} \wedge c_{3}
$$


where $c_{3}$ is the third Chern class. Thus the correction term in (3.42) is the analogue of the correction to the Einstein-Hilbert term proportional to the Euler number found for Calabi-Yau threefold compactifications in [34]. There it was shown that the kinetic terms for the moduli get a similar correction. Also in our case we expect corrections to the kinetic terms $\sim \int_{Y_{4}} d^{8} z \sqrt{\tilde{g}} E_{6}\left(Y_{4}\right)$, at least for the geometrical moduli. we have to demand that the invariants (3.43) are small for the Calabi-Yau fourfold, which in addition should have a large Euler number. In [16, 36] the invariants (3.43) are given for some examples, showing that this requirement is a rather strong constraint. We have, however, found examples in which they are considerably smaller than the Euler number.

Two further comments are in order here. First, it was found in [15] that a non-vanishing 4-form background in general necessitates generalizing the metric ansatz to a warped product. As argued in [17], the warp factor becomes trivial in the large volume limit. Here we are not considering any extended objects like space-time filling membranes or wrapped fivebranes, so the large volume limit is not obscured by the warp factor blowing up close to such sources, hence we have been working with the warp factor set to one. However, we also explicitly checked that the contributions of the warp-factor coming from the Einstein-Hilbert term and from the kinetic term of the 4-form in the compactification cancel against each other. $\square$

Second, in the presence of a 4-form flux also the expansion of the higher dimensional fields into harmonic forms, cf. (3.7), requires justification. The motivation to expand into harmonic forms in the case without flux is that they lead to zero-modes of the relevant mass operator. However, this is no longer true in the presence of fluxes. This is another way of saying that some of the former moduli become massive. Similarly to the case of the type IIB compactification on a $T^{6}$-orientifold with fluxes discussed in [38], it can be argued here that the ratio of the masses due to the flux and those of the Kaluza-Klein fields is $\left(m_{\mathrm{flux}}: m_{\mathrm{KK}}\right) \sim\left(R^{-4}: R^{-1}\right)$, with $R$ the (dimensionless) 'average' radius of the Calabi-Yau. ${ }^{18}$ Thus in the large radius limit the flux-induced masses are small compared to the Kaluza-Klein masses, and it is justified to keep only the modes coming from an expansion into the harmonic forms in the low energy effective action.

\subsection{Supersymmetry breaking}

The conditions for unbroken $N=2$ supersymmetry in a Calabi-Yau fourfold compactification with fluxes were analyzed in [15]. There it was found that the flux

\footnotetext{
${ }^{16}$ For the $(2,1)$-moduli this would require an $F_{4}^{2} R^{3}$-term.

${ }^{17}$ In a compactification of type IIB with fluxes the effects of the warp factor are discussed in 37].

${ }^{18}$ See also the discussion in $[39]$.
} 
has to be of type $(2,2)$ and primitive, i.e. $F_{4} \wedge J=0$. Taking into account (3.36) this is perfectly consistent with our general discussion (2.28).

The case of $N=1$ has not been discussed in the literature so far. The potential (3.35) is positive definite and can be written as [2]

$$
V=e^{K} G^{\alpha \bar{\beta}} D_{\alpha} W D_{\bar{\beta}} \bar{W}+8 G_{A B} D^{A} T D^{B} T
$$

with the 'covariant' derivative $D^{A} T=\partial^{A} T-\frac{1}{2}\left(\partial^{A} K\right) T$, cf. (2.22). Therefore the condition for unbroken $N=1$ can be read off from (2.30) to be

$$
2 T= \pm e^{K / 2}|W|
$$

If this is fulfilled for non-vanishing $T$ and $W$, supersymmetry is spontaneously broken to $N=1$. To be more precise, it was noted in [12, 19] that fluxes of type $F_{4} \sim J \wedge J$ or $F_{4} \sim \Omega+$ c.c. lead to a breaking of $N=2$ by generating a nonvanishing $T$ or $W$, respectively. However, the potential still vanishes. This can be understood by rewriting the potential (3.35) in yet another way [18, 2]

$$
V=-\mathcal{V}\left(\int_{Y_{4}} F_{3,1} \wedge F_{1,3}+\frac{1}{2} \int_{Y_{4}} J \wedge F_{1,1}^{(0)} \wedge J \wedge F_{1,1}^{(0)}\right),
$$

where we have used the Lefschetz decomposition of a general $(2,2)$-form flux

$$
F_{2,2}=F_{2,2}^{(0)}+J \wedge F_{1,1}^{(0)}+J^{2} \wedge F_{0,0}^{(0)}
$$

with primitive $(p, p)$-forms $F_{p, p}^{(0)}$. Thus we see that neither a flux $F_{4} \sim J \wedge J$ nor $F_{4} \sim \Omega+$ c.c. enters in the potential. Given a (locally constant) flux $F_{4}$, its expansion into $(p, 4-p)$-forms depends on the complex structure and Kähler moduli. Minimizing the potential (3.46) restricts the moduli space to the subspace, where $F_{4}$ can be expanded as

$$
\frac{F_{4}}{2 \pi}=F_{2,2}^{(0)}+F J^{2}+F_{4,0}+F_{0,4}
$$

By writing $F_{4,0}=\tilde{F} \Omega$ the condition (3.45) becomes

$$
\frac{3 F}{|\tilde{F}|}= \pm \sqrt{\frac{\int \Omega \wedge \bar{\Omega}}{\mathcal{V}}}
$$

Note that (3.45) is scale-invariant. Thus if there is one point in moduli space at which supersymmetry is broken to $N=1$, then this is true irrespective of the volume $\mathcal{V}$. This is in accordance with (3.49) despite the explicit appearance of the volume on the right hand side, because the coefficient $F$ has to scale like $\mathcal{V}^{-1 / 2}$ in order to leave the flux (3.48) constant. 


\subsection{Example}

We now give an example which makes it plausible that it is indeed possible to fulfill the condition (3.49). For simplicity we take the following ansatz for the 4 -form flux

$$
\frac{F_{4}}{2 \pi}=F J^{2}+F_{4,0}+F_{0,4}
$$

thus discarding any contribution from primitive $(2,2)$-forms. The Calabi-Yau fourfold is taken from 40. IT It is a hypersurface $X$ in a weighted projective space, whose weights can be found in the (last row of the) table of appendix A. The non-trivial Hodge numbers are given by

$$
h^{(1,1)}=7 \quad, \quad h^{(1,2)}=6 \quad, \quad h^{(1,3)}=1335 \quad, \quad h^{(2,2)}=5600 .
$$

Thus $X$ has Euler number

$$
\chi=8064
$$

The fact that the Euler number is divisible by 24 implies that $H^{4}(X, \mathbb{Z})$ is an even lattice."ण Moreover, it was shown in 41] that the flux in general has to fulfill $F_{4} / 2 \pi-p_{1} / 4 \in H^{4}(X, \mathbb{Z})$, where $p_{1}$ is the first Pontryagin class. divisible by 24 this implies $F_{4} / 2 \pi \in H^{4}(X, \mathbb{Z})$. The way we want to ensure this is by demanding that $J, \Re(\Omega)$ and $\Im(\Omega)$ are all integral themselves and by using integral coefficients $F, \tilde{F}$ in the expansion (3.50).

Thus we assume that we are at a point in moduli space of complex structure where both $\Re(\Omega)$ and $\Im(\Omega)$ are integral. As in the case at hand $H^{4}(X, \mathbb{Z})$ is an even lattice,

$$
\int_{X} \Re(\Omega) \wedge \Re(\Omega) \in 2 \mathbb{Z} \quad, \quad \int_{X} \Im(\Omega) \wedge \Im(\Omega) \in 2 \mathbb{Z} .
$$

Furthermore, because of $\int \Omega \wedge \Omega=0$ the two integrals in (3.53) are actually equal as follows from

$$
\int_{X} \Omega \wedge \Omega=\left(\int_{X} \Re(\Omega) \wedge \Re(\Omega)-\int_{X} \Im(\Omega) \wedge \Im(\Omega)\right)+2 i \int_{X} \Im(\Omega) \wedge \Re(\Omega) .(3
$$

This in turn implies that

$$
\int_{X} \Omega \wedge \bar{\Omega}=2 \int_{X} \Re(\Omega) \wedge \Re(\Omega)=4 r \in 4 \mathbb{Z} .
$$

As this integral is positive one can even be more specific: $r \in \mathbb{N}$.

\footnotetext{
${ }^{19}$ More precisely, it is in the first list of reflexive $5 \mathrm{~d}$ polyhedra.

${ }^{20}$ For a good collection of general topological properties of Calabi-Yau fourfolds see chapter 2 of [36].

${ }^{21}$ Here we make the choice $T_{2}=1$, i.e. $\kappa_{11}^{2}=2 \pi^{2}$, in order to make contact with 41].
} 
Next we choose an integral basis $e^{A}(A=1, \ldots, 7)$ for $H^{(1,1)}(X)$ which is always possible because of $h^{(2,0)}(X)=0$, i.e. $H^{2}(X, \mathbb{Z}) \otimes \mathbb{C}=H^{(1,1)}(X)$. Using the Maple packages puntos 42] and schubert [43] we calculate the volume to be

$$
\begin{aligned}
\mathcal{V}= & 6 M_{2} M_{3} M_{4} M_{5}+\frac{8}{3} M_{4} M_{5}^{3}+\frac{4}{3} M_{2} M_{5}^{3}+6 M_{3} M_{4} M_{5}^{2} \\
& +12 M_{3} M_{5} M_{4}^{2}+8 M_{3} M_{6}^{3}+\frac{8}{3} M_{5} M_{4}^{3}+2 M_{1} M_{2} M_{5} M_{6} \\
& +2 M_{1} M_{2} M_{6}^{2}+6 M_{1} M_{5}^{2} M_{6}+4 M_{1} M_{5} M_{6}^{2}+\frac{8}{3} M_{1} M_{6}^{3} \\
& +6 M_{1} M_{7}^{3}+2 M_{2} M_{4} M_{5}^{2}+3 M_{5}^{2} M_{6}^{2}+\frac{2}{3} M_{1}^{3} M_{7}+3 M_{2} M_{7}^{3} \\
& +\frac{8}{3} M_{5} M_{6}^{3}+12 M_{3} M_{5} M_{6}^{2}+18 M_{3} M_{5}^{2} M_{6}+2 M_{6}^{4}+\frac{4}{3} M_{2} M_{6}^{3} \\
& +6 M_{2} M_{3} M_{5} M_{6}+M_{2} M_{5} M_{6}^{2}+6 M_{2} M_{3} M_{6}^{2}+2 M_{1} M_{2} M_{4} M_{5} \\
& +5 M_{5}^{2} M_{4}^{2}+\frac{9}{2} M_{7}^{4}+6 M_{2} M_{3} M_{5}^{2}+\frac{2}{3} M_{5}^{4}+M_{2} M_{5} M_{4}^{2} \\
& +\frac{2}{3} M_{4}^{4}+\frac{5}{6} M_{1}^{4}+\frac{32}{3} M_{1}^{3} M_{3}+48 M_{1}^{2} M_{3}^{2}+12 M_{1} M_{2} M_{3}^{2} \\
& +8 M_{3} M_{4}^{3}+2 M_{1} M_{4} M_{5}^{2}+4 M_{1} M_{5} M_{4}^{2}+M_{1}^{2} M_{2} M_{7} \\
& +3 M_{1} M_{2} M_{7}^{2}+3 M_{1}^{2} M_{7}^{2}+8 M_{3} M_{5}^{3}+\frac{8}{3} M_{1} M_{5}^{3}+2 M_{1} M_{2} M_{5}^{2} \\
& +4 M_{1}^{2} M_{2} M_{3}+\frac{1}{3} M_{1}^{3} M_{2}+96 M_{1} M_{3}^{3}+72 M_{3}^{4}+12 M_{2} M_{3}^{3} \\
& +2 M_{1} M_{2} M_{4}^{2}+\frac{8}{3} M_{1} M_{4}^{3}+\frac{4}{3} M_{2} M_{4}^{3}+6 M_{2} M_{3} M_{4}^{2} .
\end{aligned}
$$

Now we have to make sure that both the condition (3.49) and the tadpole condition (3.5) are fulfilled at the same time. For the choice $T_{2}=1$ that we use for this example (see footnote 21) (3.5) takes the form

$$
\frac{1}{4 \pi^{2}} \int_{Y_{4}} F_{4} \wedge F_{4}=\frac{\chi}{12} .
$$

Inserting $\frac{F_{4}}{2 \pi}=F J^{2}+(\tilde{F} \Omega+c . c)$ and using (3.49) leads to the two conditions

$$
F^{2}=\frac{\chi}{504 \mathcal{V}} \quad, \quad|\tilde{F}|^{2}=\frac{\chi}{224 r} .
$$

Assuming $r=1$ and choosing the Kähler moduli

$$
M_{1}=2 \quad, \quad M_{2}=1 \quad, \quad M_{3}=\ldots=M_{7}=0,
$$

leading to a volume of $\mathcal{V}=16$ and an integral Kähler form, we see that for the value of the Euler number (3.52) these two conditions can be satisfied by

$$
F=1 \quad, \quad \tilde{F}=6 .
$$

Thus we see that at this special point in moduli space, the flux

$$
\frac{F_{4}}{2 \pi}=J \wedge J+12 \Re(\Omega)
$$

is in $H^{4}(X, \mathbb{Z})$, fulfills the tadpole condition and breaks supersymmetry from $N=2$ to $N=1$. 
Strictly speaking the values (3.58) for the Kähler moduli are outside the range of validity of our effective theory (which is at large volume), because some of the Kähler moduli vanish. As this means that some of the two-cycles of the CalabiYau shrink to zero size, one generically expects new light degrees of freedom from membranes wrapped around these vanishing cycles. This problem is due to our special Ansatz for $F_{4}$. To ensure integrality of $\frac{F_{4}}{2 \pi}$, we demanded that $J, \Re(\Omega)$ and $\Im(\Omega)$ are all integral themselves. Relaxing this requirement would certainly allow for partial supersymmetry breaking within the range of large volume.

Let us look at bit closer at this issue. Giving up the demand for an integral $J$ and requiring only $F J^{2} \in H^{4}(X, \mathbb{Z})$, shows that one is free to rescale all Kähler

moduli by a common factor $\sqrt{\lambda}$ (and thus rescaling the volume $\mathcal{V}$ by $\lambda^{2}$ ) if one rescales $F$ by $\lambda^{-1}$ at the same time. This corresponds to the observation made below (3.49). However, even then some of the Kähler moduli remain at zero. Thus to avoid this problem one should give up also the requirement that $F J^{2}$ and $(\tilde{F} \Omega+$ c.c. $)$ are separately in $H^{4}(X, \mathbb{Z})$, a situation which is hard to analyze explicitly.

\section{Acknowledgments}

We are grateful for useful discussions with B. Körs, S. Hellerman and M. Schulz. We would especially like to thank V. Braun and J. de Loera for essential help with the Maple packages puntos and schubert.

This work was supported in part by I.N.F.N., by the EC contract HPRN-CT2000-00122, by the EC contract HPRN-CT-2000-00148, by the INTAS contract 99-0-590 and by the MURST-COFIN contract 2001-025492. M.B. was supported by a Marie Curie Fellowship, contract number HPMF-CT-2001-01311.

\section{A Search for an example}

To satisfy the $N=1$ condition (3.49) with $r$ in (3.55) being unity, we need a Calabi-Yau fourfold with an Euler number $\chi$ divisible by 24 and 224, and a volume $\mathcal{V}$ which is such that $\chi /(504 \mathcal{V})$ is a square integer for some values of the Kähler moduli (cf. (3.57)). For example, for $\chi=2016$, this means $\mathcal{V}$ can be 1 or 4 . If none of these special volume values can be obtained for integral Kähler moduli $M_{A}$, the Calabi-Yau manifold does not satisfy the $N=1$ condition (3.49) given tadpole cancellation and the integrality assumptions of section 3 .

Now, in the tables 40 of Calabi-Yau fourfold hypersurfaces in toric varieties, the Euler number and a few other properties are explicitly given. To compute the volume $\mathcal{V}$ in terms of the Kähler moduli, one also needs intersection numbers of the corresponding divisors, and for this one needs to desingularize the toric variety. This is done by triangulating the corresponding polytope (see for instance [44] for a review). Triangulating our way through the tables [40] in order of increasing number of points in the polytope and increasing $h^{(1,1)}$, it turns out that three of the 
candidate toric varieties we tried (indicated in table 1) cannot be desingularized; fractional intersection numbers appear. In the other five cases, the toric variety can be made smooth, and in the last candidate in the table, the condition (3.49) can, in fact, be satisfied together with tadpole cancellation and integrality conditions — for the values in (3.58) only.

\begin{tabular}{|c|c|c|c|c|c|c|c|c|c|c|c|}
\hline \multicolumn{4}{|c|}{ weights } & degree & $\mathrm{M}$ & $\mathrm{N}$ & $h^{(1,1)}, h^{(1,2)}, h^{(1,3)}$ & $\chi$ & comment \\
\hline \hline 1 & 2 & 3 & 3 & 3 & 6 & 18 & 354 & 9 & $3,0,325$ & 2016 & singular \\
\hline 1 & 2 & 4 & 7 & 7 & 7 & 28 & 367 & 10 & $4,9,333$ & 2016 & singular \\
\hline 1 & 1 & 1 & 1 & 4 & 7 & 15 & 1525 & 10 & $4,0,1332$ & 8064 & \\
\hline 1 & 1 & 2 & 2 & 6 & 12 & 24 & 1547 & 10 & $4,0,1332$ & 8064 & \\
\hline 2 & 2 & 2 & 2 & 7 & 15 & 30 & 1021 & 13 & $4,0,1332$ & 8064 & \\
\hline 1 & 2 & 2 & 2 & 8 & 15 & 30 & 1461 & 14 & $5,0,1331$ & 8064 & \\
\hline 1 & 2 & 2 & 6 & 10 & 21 & 42 & 1483 & 12 & $4,0,1332$ & 8064 & singular \\
\hline 1 & 1 & 6 & 8 & 8 & 24 & 48 & 1547 & 13 & $7,6,1335$ & 8064 & $N=1$ \\
\hline
\end{tabular}

Table 1: Some Calabi-Yau hypersurfaces in weighted $\mathbb{P}^{n_{1}, \ldots n_{6}}$, with Euler numbers divisible by 24, 224 and 504, taken from the tables of [40]. The number of points in the $\mathrm{M}$ and $\mathrm{N}$ lattices are given; the N-lattice polytope is the one that is triangulated.

\section{References}

[1] S.B. Giddings, S. Kachru and J. Polchinski, "Hierarchies from fluxes in string compactifications", hep-th/0105097.

[2] K. Becker, M. Becker, M. Haack and J. Louis, "Supersymmetry breaking and alpha' corrections to flux induced potentials", JHEP 0206 (2002) 060, hepth/0204254.

[3] C. Angelantonj and A. Sagnotti, "Open strings", Phys. Rept. 371 (2002) 1, hep-th/0204089.

[4] J. Michelson, "Compactifications of type IIB strings to four dimensions with non-trivial classical potential", Nucl. Phys. B495 (1997) 127, hep-th/9610151.

[5] T.R. Taylor and C. Vafa, "RR flux on Calabi-Yau and partial supersymmetry breaking", Phys. Lett. B474 (2000) 130, hep-th/9912152.

[6] P. Mayr, "On supersymmetry breaking in string theory and its realization in brane worlds", Nucl. Phys. B593 (2001) 99, hep-th/0003198.

[7] G. Curio, A. Klemm, D. Lüst and S. Theisen, "On the vacuum structure of type II string compactifications on Calabi-Yau spaces with H-fluxes", Nucl. Phys. B609 (2001) 3, hep-th/0012213. 
[8] G. Dall'Agata, "Type IIB supergravity compactified on a Calabi-Yau manifold with H-fluxes", JHEP 0111 (2001) 005, hep-th/0107264.

[9] J. Louis and A. Micu, "Type II theories compactified on Calabi-Yau threefolds in the presence of background fluxes", Nucl. Phys. B635 (2002) 395, hepth/0202168.

[10] C. Vafa, "Evidence for F-Theory", Nucl. Phys. B469 (1996) 403, hepth/9602022.

[11] A. Sen, "F-theory and Orientifolds", Nucl. Phys. B475 (1996) 562, hepth/9605150.

[12] S. Gukov, C. Vafa and E. Witten, "CFT's from Calabi-Yau four-folds", Nucl. Phys. B584 (2000) 69, Erratum-ibid. B608 (2001) 477, hep-th/9906070.

[13] K. Dasgupta, G. Rajesh and S. Sethi, "M theory, orientifolds and G-flux", JHEP 9908 (1999) 023, hep-th/9908088.

[14] B. de Wit, I. Herger and H. Samtleben, "Gauged locally supersymmetric $D=$ 3 nonlinear sigma models," to appear.

[15] K. Becker and M. Becker, "M-theory on eight-manifolds", Nucl. Phys. B477 (1996) 155, hep-th/9605053.

[16] P. Mayr, "Mirror symmetry, N=1 superpotentials and tensionless strings on Calabi-Yau fourfolds", Nucl. Phys. B494 (1997) 489, hep-th/9610162.

[17] B.R. Greene, K. Schalm and G. Shiu, "Warped compactifications in M and F theory", Nucl. Phys. B584 (2000) 480, hep-th/0004103.

[18] M. Haack and J. Louis, "M-theory compactified on Calabi-Yau fourfolds with background flux", Phys. Lett. B507 (2001) 296, hep-th/0103068.

[19] K. Becker and M. Becker, "Supersymmetry breaking, M-theory and fluxes", JHEP 0107 (2001) 038, hep-th/0107044.

[20] E. Witten, "Strong coupling and the cosmological constant", Mod. Phys. Lett. A10 (1995) 2153, hep-th/9506101.

[21] M. Haack and J. Louis, "Duality in heterotic vacua with four supercharges", Nucl. Phys. B575 (2000) 107, hep-th/9912181.

[22] N.S. Deger, A. Kaya, E. Sezgin and P. Sundell, "Matter coupled $\mathrm{AdS}_{3}$ supergravities and their black strings," Nucl. Phys. B573 (2000) 275, hepth/9908089. 
[23] M. Abou-Zeid and H. Samtleben, "Chern-Simons vortices in supergravity," Phys. Rev. D65 (2002) 085016, hep-th/0112035.

[24] P. Binétruy, G. Girardi and R. Grimm, "Supergravity couplings: A geometric formulation", Phys. Rept. 343 (2001) 255, hep-th/0005225.

[25] P. Adamietz, P. Binétruy, G. Girardi and R. Grimm, "Supergravity and matter: Linear multiplet couplings and Kähler anomaly cancellation", Nucl. Phys. B401 (1993) 257.

[26] E. Cremmer, B. Julia and J. Scherk, "Supergravity theory in elevendimensions", Phys. Lett. B76 (1978) 409.

[27] C. Vafa and E. Witten, "A one loop test of string duality", Nucl. Phys. B447 (1995) 261, hep-th/9505053.

[28] M.J. Duff, J.T. Liu and R. Minasian, "Eleven-dimensional origin of stringstring duality: A one loop test", Nucl. Phys. B452 (1995) 261, hepth/9506126.

[29] S. Sethi, C. Vafa and E. Witten, "Constraints on low dimensional string compactifications", Nucl. Phys. B480 (1996) 213, hep-th/9606122.

[30] K. Dasgupta and S. Mukhi, "A note on low-dimensional string compactifications", Phys. Lett. B398 (1997) 285, hep-th/9612188.

[31] M. Haack, J. Louis and M. Marquart, "Type IIA and heterotic string vacua in D =2", Nucl. Phys. B598 (2001) 30, hep-th/0011075.

[32] K. Peeters, P. Vanhove and A. Westerberg, "Supersymmetric higher-derivative actions in ten and eleven dimensions, the associated superalgebras and their formulation in superspace", Class. Quant. Grav. 18 (2001) 843, hepth/0010167.

[33] A.A. Tseytlin, " $R^{4}$ terms in 11 dimensions and conformal anomaly of $(2,0)$ theory", Nucl. Phys. B584 (2000) 233, hep-th/0005072.

[34] I. Antoniadis, S. Ferrara, R. Minasian and K.S. Narain, " $R^{4}$ couplings in $M$ and type II theories on Calabi-Yau spaces", Nucl. Phys. B507 (1997) 571, hep-th/9707013.

[35] D. Gross and E. Witten, "Superstring modification of Einstein equations", Nucl. Phys. B277 (1986) 1.

[36] A. Klemm, B. Lian, S.S. Roan and S.T. Yau, "Calabi-Yau fourfolds for M- and F-theory compactifications", Nucl. Phys. B518 (1998) 515, hep-th/9701023. 
[37] O. DeWolfe and S.B. Giddings, "Scales and hierarchies in warped compactifications and brane worlds", hep-th/0208123.

[38] S. Kachru, M.B. Schulz and S. Trivedi, "Moduli stabilization from fluxes in a simple IIB orientifold", hep-th/0201028.

[39] N. Kaloper and R.C. Myers, "The O(dd) story of massive supergravity", JHEP 9905 (1999) 010, hep-th/9901045.

[40] M. Kreuzer and H. Skarke, "Calabi-Yau data, 4-folds and beyond: Weight systems in 5 and 6 dimensions", http://hep.itp.tuwien.ac.at/ kreuzer/CY].

[41] E. Witten, "On flux quantization in M-theory and the effective action", J. Geom. Phys. 22 (1997) 1, hep-th/9609122.

[42] http://www.math.ucdavis.edu/ deloera/

[43] http://www.math.okstate.edu/ katz/schubert.htm]

[44] B.R. Greene, "String theory on Calabi-Yau manifolds", hep-th/9702155. 